\title{
Unidirectional Light Propagation in Natural Crystals
}

0 ne-way propagation of light is not a phenomenon expected in everyday contexts, such as glass windows and sunlight. Nevertheless, the possibility of one-way propagation is the basis for a variety of optical applications, ranging from signal-processing devices for communication to whimsical examples such as Harry Potter-style invisibility cloaks. In the case of invisibility cloaks, one-way light propagation is essential for concealment, and studies of ways to achieve this through synthetic materials or metamaterials are ongoing. 1

In recent work, we showed that one-way light propagation, with respect to the incidence angle, can occur in simple, natural crystalline materials, such as crystal quartz, at far-infrared frequencies. ${ }^{2}$ While such an optical response in natural crystalline materials seems counterintuitive, these materials can have remarkable electromagnetic properties at particular frequencies, known as resonances.

These resonances are direction dependent ${ }^{3}$ and, close to the resonance frequencies, metamaterial-like optical behavior can arise, including negative refraction, due to hyperbolic dispersion-that is, the principal components of the permittivity tensor possess opposite signs. Moreover, by controlling these resonances through modifying the anisotropy direction with respect to the crystal surface, we can create or destroy regions in which light propagation is possible. This effectively can create a "two-way" material, making it opaque to light incident from one side while transparent to light incident from the other.

In experiments conducted with these materials, we found that at frequencies close to $550 \mathrm{~cm}^{-1}$ (equivalent to a wavelength of $18 \mu \mathrm{m}$ ) and negative angles of incidence, maximum absorption is observed, whereas for positive incidence angles, absorption is close to a minimum. Our results support the idea that natural crystals can serve as efficient and functional oriented asymmetric absorbers. ${ }^{2}$ These features, together with their widespread availability, have led to the use of materials such as the quartz we have studied in developing on-chip devices. ${ }^{4}$ OPN

\section{RESEARCHERS}

R. Macêdo (Rair.Macedola glasgow.ac.uk), C.A. McEleney and M. González-Jiménez.

University of Glasgow,

Glasgow, U.K.

$\mathbf{X}$. Wu, Shandong Institute of Advanced Technology. Jinan, China

\section{REFERENCES}

1. X. Yin and $X$. Zhang. Nat. Mater. 12, 175 (2013).

2. X. Wu et al. Optica 6, 1478 (2019).

3. R. Macêdo et al. ACS Photon. 5, 5086 (2018)

4. L. Shen et al. Appl. Phys. Rev. 7, 021403 (2020).
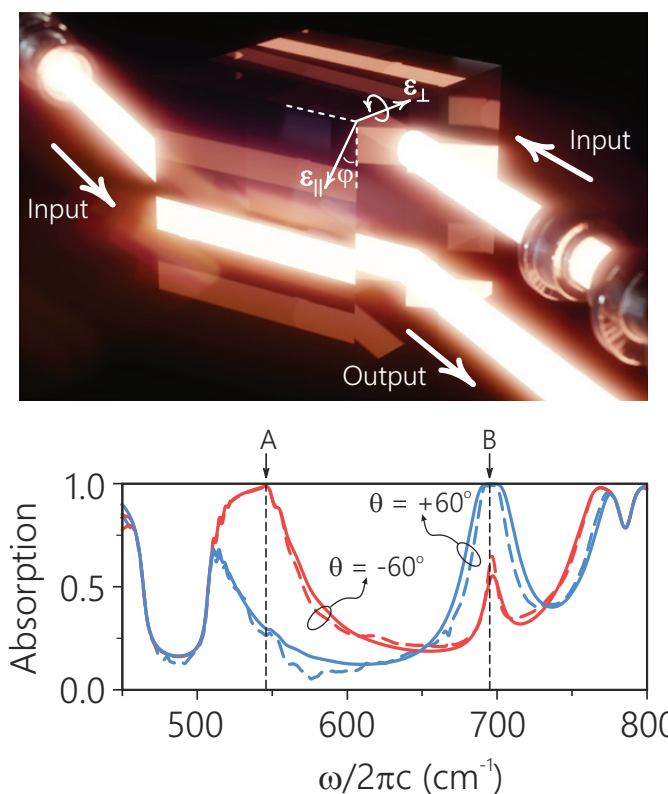

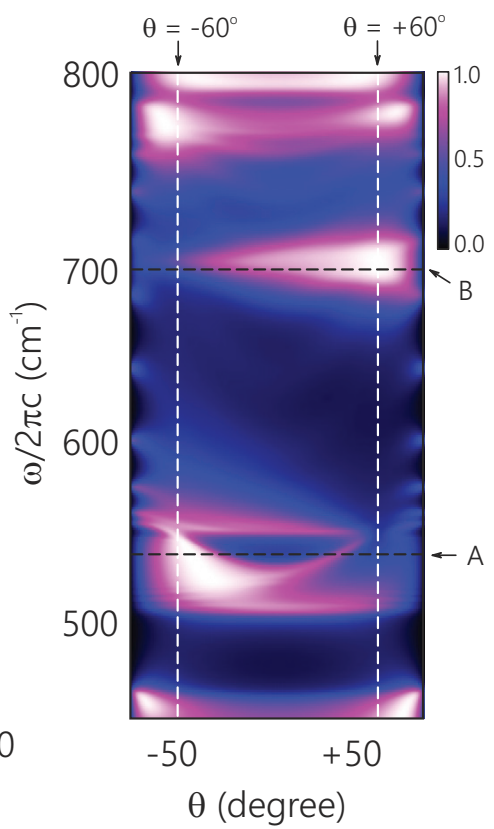

$\theta$ (degree)

Unidirectional propagation in crystalline quartz. Top left: Illustration of a beam incident from two different orientations. Bottom left: Theoretical (solid lines) and experimental (dashed lines) absorption spectra for positive (blue lines) and negative (red lines) incident beam. Right: Summary map of the absorption as a function of incidence angle and frequency. 